\title{
Promoção em saúde mental de adolescentes em países da América Latina: uma revisão integrativa da literatura
}

\author{
Adolescent mental health promotion in Latin American countries: \\ an integrative literature review
}

Thaís Thaler Souza (https://orcid.org/0000-0001-6623-4975) ${ }^{1}$

Ana Carolina de Almeida (https://orcid.org/0000-0001-9449-2365) ${ }^{2}$

Amanda Dourado Souza Akahosi Fernandes (http://orcid.org/0000-0001-8006-8117) ${ }^{3}$

Maria Fernanda Barboza Cid (https://orcid.org/0000-0002-0199-0670) ${ }^{4}$

${ }^{1}$ Programa de Pós-

Graduação em Terapia Ocupacional, Universidade Federal de São Carlos (UFSCar). Rod. Washington Luiz, km 235. 13565-905 São Carlos SP Brasil. thaisthaler@hotmail.com

${ }^{2}$ Graduação em Terapia

Ocupacional, UFSCar. São

Carlos SP Brasil.

${ }^{3}$ Departamento de Terapia Ocupacional. UFSCar. São

Carlos SP Brasil.

${ }^{4}$ Programa de Pós-

Graduação em Educação

Especial, UFSCar. São

Carlos SP Brasil.

\begin{abstract}
Health Promotion (HP) has recently been strengthened within public policies and still shows strong consonance with international determinations regarding childhood and adolescence in Latin America (LA). Debating HP becomes all the more urgent considering the increasing prevalence of psychological distress in this age group and the historical political construction of Latin American countries. This research aimed to identify and analyze, through an integrative review of the literature, the academic productions on strategies for mental HP of adolescents in LA and, thus, to visualize the actions and arouse reflections dialoguing with the Southern critics, represented by the Bogotá declaration. Six studies were selected after applying the inclusion and exclusion criteria. We identified that the main space where actions are developed is the school. The potential measures identified were professional training, group action, and strengthening primary and territorial care. We discussed that the historically structured socioeconomic reality of LA reflects an organization of HP actions. Furthermore, the visualization of strategies that enhance at low cost the improved mental health of adolescents can contribute to the current reflection.
\end{abstract}

Key words Health Promotion, Adolescents, Latin America
Resumo A Promoção em Saúde (PS) apresenta um fortalecimento recente dentro das políticas públicas e quando se trata da infância e adolescência na América Latina (AL) observa-se ainda forte consonância com as determinações internacionais. Considerando o aumento de prevalência de sofrimento psíquico nesta faixa etária e a construção histórica política dos países latino-americanos abordar a PS se torna mais urgente. O objetivo desta pesquisa foi identificar e analisar, por meio de uma revisão integrativa da literatura, produções acadêmicas que tratam de estratégias de PS mental de adolescentes na AL e, assim, visibilizar as ações e provocar reflexões dialogadas com as críticas Sulistas, representadas pela declaração de Bogotá. Após a aplicação dos critérios de inclusão e exclusão, foram selecionados 6 estudos. Identificou-se que o principal espaço onde as ações são desenvolvidas é a escola. Além disso, como medidas potenciais foram identificadas a capacitação dos profissionais, a ação grupal e o fortalecimento da atenção primária e territorial. Discute-se que a realidade socioeconômica estruturada historicamente da AL reflete uma organização própria das ações em PS. Com isso, a visibilização das estratégias que potencializam a baixo custo uma melhora na saúde mental de adolescentes pode contribuir com a reflexão atual.

Palavras-chave Promoção da Saúde, Adolescente, América Latina 


\section{Introdução}

O conceito de Promoção em Saúde (PS) e suas práticas começaram a ser mais fortemente difundidas a partir da década de 1980. Atrelada ao "desenvolvimento sustentável" e "políticas públicas saudáveis", a PS rapidamente foi correlacionada aos fatores ambientais, sociais, psicológicos, físicos, sendo resultante das condições de vida. A retomada desse conceito nos últimos 40 anos, aconteceu pela iniciativa de países da Europa, Canadá, Estados Unidos e tinha como objetivo a redução dos altos custos em saúde que o modelo biomédico promove, além de uma crítica quanto a eficiência do sistema vigente ${ }^{1}$.

No entanto, de acordo com Spink ${ }^{2}$, em decorrência da própria estrutura político-social que o capitalismo gera, e sua característica de incentivo ao individualismo como modo de sobrevivência, a forma com que a PS é conceituada nesses últimos anos tem apresentado uma variação de acordo com interesses locais e mercantis, influenciando, inclusive, na quantidade de interferência Estatal que se deve ou não ter em ações dessa natureza, do "dever" em saúde dos sujeitos e os objetivos que se tem com a utilização dessa prática. Ou seja, o respaldo e o incentivo a PS apresentam uma variabilidade, que vai desde vertentes mais conservadoras, com um movimento histórico de diminuição do papel do Estado e aumento de responsabilização do sujeito sobre seu próprio processo de saúde, até perspectivas mais progressistas de movimentos reflexivos e de resistências dessas construções com uma maior cobrança de suporte de medidas de políticas públicas ${ }^{1,2}$.

$\mathrm{Na}$ América Latina (AL), com a Medicina Social, e no Brasil, com a Saúde Coletiva, têm se desenvolvido uma perspectiva fortemente crítica do conceito. Falar de PS na AL remete a reflexões sobre conceituações que vieram junto com os processos de colonização e estimula a questionar possibilidades que são mais compatíveis com um contexto político marcado por vulnerabilidades de diferentes ordens ${ }^{3}$.

Freitas ${ }^{4}$ sinaliza que se a publicação da Carta de Ottawa no Canadá em 1986 apresenta-se como o documento referência para as ações de promoção em Saúde no mundo, para a AL a declaração de Bogotá, 1992, trouxe reflexões importantes sobre a discussão de PS vinculada a práticas que sejam contextualizadas e que auxiliem nos processos de transformação social. Em suma, a declaração de Bogotá enfatiza sobre a importância de quebra das estruturas que mantêm o status quo de desigualdade, com o incentivo de ações democráticas, equitativas e inovadoras".
A resistência dos países da AL em relação a essa opressão histórica de impor conceitos como, o que é ser desenvolvido, o padrão de bem-estar e viver bem, fez surgir uma potência criadora marginalizada. Com isso, de acordo com Acosta ${ }^{5}$ essas perspectivas pós-desenvolvimentistas dos países latinos objetivam ir para além de "desenvolvimentos alternativos" e visam possibilitar "alternativas de desenvolvimento". Desta forma, atualmente, o conceito de PS:

[...] está associado inicialmente a um 'conjunto de valores': vida, saúde, solidariedade, equidade, democracia, cidadania, desenvolvimento, participação e parceria, entre outros. Refere-se também a uma 'combinação de estratégias': ações do Estado (políticas públicas saudáveis), da comunidade (reforço da ação comunitária), de indivíduos (desenvolvimento de habilidades pessoais), do sistema de saúde (reorientação do sistema de saúde) e de parcerias intersetoriais; isto é, trabalha com a ideia de 'responsabilização múltipla', seja pelos problemas, seja pelas soluções propostas para os mesmos. ${ }^{1}$ (p.20).

Apesar da influência "internacionalista", ainda bastante presente no conceito, e possível de ser identificada nas conferências internacionais, determinações políticas, cartas e outras instâncias políticas, tem se observado um movimento de valorização dos saberes culturais e de participação social e promoção de parcerias com movimentos sociais envolvidos com o meio ambiente e o feminismo, por exemplo ${ }^{1}$.

Contudo, quando se trata de PS para a adolescência ainda se identifica expressivo atravessamento das determinações internacionais e das vantagens econômicas de investimento desta natureza com este público. No manual produzido pela Organização Mundial de Saúde ${ }^{6}$, em parceria com outros órgãos das Nações Unidas, que fornece informações abrangentes sobre a saúde dos adolescentes para diversos países, são listados os motivos da necessidade de se investir na saúde do adolescente.

Segundo o documento ${ }^{6}$, a PS apresenta vantagens para a saúde imediata dos adolescentes e contribui para esse estado mesmo na idade adulta, reverberando para as gerações futuras. O documento declara ainda, que o investimento em promoção de saúde para crianças e adolescentes corresponde a máxima dos direitos humanos, a qual quase todos os países são signatários. Os benefícios econômicos e demográficos da prática de PS para adolescentes apresentam-se de maneira destacada no texto ${ }^{6}$, e são justificados com o aumento da produtividade, da redução dos custos em saúde e o consequente aumento de capital social. 
Em relação às principais demandas de saúde dos adolescentes, considera-se que a saúde mental tem sido tema emergente na atualidade, uma vez que a prevalência do sofrimento psíquico nessa população tem aumentado significativamente nos últimos anos ${ }^{7,8}$. Nessa direção, estudos sinalizam para a necessidade do investimento em políticas públicas, pesquisas e estratégias de cuidado que visem a promoção à saúde mental dos adolescentes, principalmente quando se trata de países em desenvolvimento?.

Porém, identifica-se na literatura que a produção científica de saúde mental em países de baixo e médio rendimento representam apenas cerca de $6 \%$ desse conteúdo, sendo este número ainda menor quando especificada para saúde mental de crianças e adolescentes ${ }^{10}$ e quando pensada em uma perspectiva mais salutogênica do problema, voltadas para a promoção e prevenção, encontramos um campo ainda em construção ${ }^{11}$.

A título de exemplo, em um estudo realizado em Unidades básicas de Saúde do Estado de São Paulo por Fernandes ${ }^{12}$, identificou-se que as ações de promoção à saúde mental infantojuvenis ainda são distantes das práticas de cuidado ofertadas, mesmo considerando os territórios de maior vulnerabilidade social, onde a pobreza, tráfico de drogas, violência se fazem presentes. Assim, os resultados sinalizam que o cuidado a essa população tem se pautado exclusivamente na perspectiva dos problemas de saúde, pouco considerando os determinantes sociais, os quais estão diretamente relacionados às condições de saúde, de forma que estar em um contexto de vulnerabilidade significaria aumentar os riscos e as chances de comprometimento no desenvolvimento das crianças e adolescentes ${ }^{12,13}$.

Nessa direção, considerando o fortalecimento recente do conceito de PS no mundo e a, ainda mais recente, construção crítica em torno da ideia nos países da $\mathrm{AL}^{1}$ reforçam a importância de refletir sobre o que tem sido proposto e desenvolvido aos adolescentes, uma vez que quando se trata de saúde mental infantojuvenil, sua contemporaneidade e sua conceituação, ainda em construção, deparamo-nos com atravessamentos de ordem política, de uma negação histórica e de não valorização e reconhecimento desses sujei$\operatorname{tos}^{14}$.

Com isso, o presente estudo pretende contribuir, na medida em que se propõe a identificar e analisar, por meio de uma revisão integrativa da literatura, produções acadêmicas que tratam de estratégias de promoção à saúde mental de adolescentes na AL, tendo em vista, o avanço na construção de conhecimento que esteja condizente com o contexto histórico-social desta região do continente americano, marcado por sua curvatura populacional jovem e de vulnerabilidade e, assim, visibilizar as ações e provocar reflexões dialogadas com as críticas Sulistas, representadas pela declaração de Bogotá ${ }^{4,9}$.

\section{Método}

Trata-se de uma revisão integrativa da literatura, a qual configura-se enquanto um método de pesquisa que possibilita a síntese e análise do que existe de produção sobre determinado fenômeno. Além disso, tem como objetivo a produção de novos questionamentos, reflexões e críticas, auxiliando na identificação de lacunas existentes e, consequentemente, no avanço do conhecimento ${ }^{15}$. Os seguintes passos da revisão integrativa foram seguidos pelo presente estudo: 1) Identificação da questão de pesquisa; 2)Busca na literatura científica (para esta etapa foram estabelecidos critérios de inclusão e exclusão para o material encontrado, realização da busca nas bases de dados e seleção estudos); 3 ) Categorização dos resultados encontrados; 4) Avaliação dos artigos selecionados; 5) Análise, interpretação e discussão dos resultados; e 6) Sintetização das informações e produção de conhecimento ${ }^{16}$.

Para tanto, foi formulada a seguinte questão de pesquisa: $\mathrm{O}$ que a literatura tem produzido sobre as estratégias de promoção à saúde mental de adolescentes na América Latina?

A busca foi realizada nas seguintes bases de dados: Biblioteca Virtual em Saúde (BVS), Scopus e Web of Science, no período de setembro de 2019 a abril de 2020. A escolha pelas bases de dados teve como objetivo acessar o máximo do conteúdo dos países ibero-americanos. Os descritores foram selecionados a partir dos Descritores em Ciências da Saúde (DECS) em inglês, espanhol e português para a BVS, e para as demais bases, os descritores foram identificados na Medical Subject Headings (MeSH) e buscados em inglês e espanhol. Os descritores identificados no DECS e MesSH coincidiram, sendo eles: "saúde mental", "promoção de saúde", adolescen*, e nas suas variações em inglês e espanhol: "mental health"/ "salud mental"; adolescen*; e "health promotion/ promoción de la salud".

Os descritores foram combinados da seguinte forma: ("promoção de saúde" OR "health promotion"OR promoción de la salud") AND ("saúde 
mental" OR "mental health" OR "salud mental") AND (adolescen ${ }^{*}$ ) para BVS, e para as demais bases ("health promotion OR promoción de la salud") AND ("mental health" OR "salud mental") AND (adolescen ${ }^{*}$.

Os seguintes critérios de inclusão foram adotados para a seleção das produções: artigos em inglês, espanhol e português, que explicitassem em título, resumo e palavras chaves qualquer tipo de estratégia, ação e/ou programa de promoção em saúde mental para o público adolescente. Após a seleção dos estudos, ainda nas bases de dados, foram selecionados por meio do filtro "países e territórios" apenas os países da América Latina. Por outro lado, artigos que não estivessem disponibilizados integralmente online, revisões de literatura, levantamentos epidemiológicos, correspondências, editoriais, monografias, dissertações e teses foram produções excluídas da presente revisão. Além disso, cabe apontar que não foi adotado nenhum recorte temporal para a busca. Na Figura 1, ilustra-se o fluxograma seguido pelo presente estudo.

Para análise dos artigos selecionados, após a leitura criteriosa na íntegra dos mesmos, foi realizada uma análise descritiva dos materiais, considerando as variáveis: ano e país da produção, referencial teórico utilizado, objetivo do trabalho publicado, métodos e principais resultados encontrados. Tal análise resultou na configuração de eixos temáticos que serão apresentados na próxima seção.

\section{Resultados}

Nos Quadros 1 e 2 contêm informações relativas aos 6 artigos que compõem a amostra final selecionada para o presente estudo.

Identifica-se no Quadro 1 que os estudos identificados na presente revisão foram desenvolvidos em três países da $\mathrm{AL}$ - três deles no Brasil $^{17-19}$, dois no Chile ${ }^{20,21}$ e um na Colômbia ${ }^{22}$. Quanto ao ano de publicação, cinco deles são da última década ${ }^{17-20,22}$ e apenas um do ano de $2005^{21}$. Quanto às áreas de produção, observa-se que quatro publicações são da área de saúde pública e coletiva.

Em relação ao tipo de estudo, foi observado, principalmente nas produções não brasileiras, uma metodologia de pesquisa com predomínio do tipo experimental e quase-experimental ${ }^{20-22}$.

Em relação a fundamentação teórica adotada, em quatro dos artigos selecionados, verifica-se embasamento na literatura que trata do fortale- cimento de habilidades individuais de enfrentamento baseado no coping e estratégias cognitivas e comportamentais ${ }^{17,20-22}$. Os outros dois estudos são fundamentados pela perspectiva da educação em saúde, sendo ambos brasileiros ${ }^{18,19}$.

Quanto aos participantes da pesquisa, apenas um artigo contemplou a comunidade e os pais nas construções das ações de promoção, além dos profissionais e adolescentes ${ }^{17}$, e dois abarcaram somente os profissionais da Saúde e/ou Educação $^{19,22}$. As demais produções realizaram intervenções com um enfoque unicamente pautado nos adolescentes ${ }^{17,18,20,21}$.

Destaca-se, ainda, que três estudos ${ }^{17,19,22}$ focalizaram a formação dos profissionais que trabalham com adolescentes como estratégia de PS com este público: um artigo realizou um treinamento com professores na modalidade à distância e presencial, voltado para identificação de sintomatologias dentro do sofrimento mental ${ }^{22}$, um realizou treinamento dos professores para trabalhar com adolescentes em relação a álcool e outras drogas e um trabalhou com profissionais de saúde de forma a construir possibilidades de melhor assistência ao atendimento do adolescente na saúde mental ${ }^{19}$.

O Quadro 3 apresenta os seis eixos temáticos identificados a partir dos principais resultados dos estudos.

Apesar da busca pela parceria com o contexto escolar na quase totalidade dos estudos, quatro dos artigos selecionados tiveram dificuldades no trabalho em rede e intersetorial ${ }^{17-20}$. Dentre esses impasses é citado o não apoio dos gestores da escola, o que acabou provocando um "desânimo" na participação dos professores que tinham dificuldade em manter o cronograma obrigatório, os autores citam ainda, sobre uma possível postura verticalizada por parte da saúde em relação ao saber técnico-científico não realizando uma real parceria com outras instâncias e comunidade. São relatadas, também, dificuldades em relação ao estabelecimento de parcerias dentro do território, desarticulações na assistência, fragilidade na relação com a comunidade e baixa parceria com os pais e responsáveis ${ }^{17-20}$.

A potência da estratégia de utilização de grupos com os adolescentes é citada em três dos artigos selecionados ${ }^{18,20,21}$, cujos autores sugerem ser uma possibilidade metodológica interessante para esta faixa-etária. A formação dos profissionais que trabalham com os adolescentes também foi um fator apontado como potente nos resultados dos estudos analisados, sendo identificado em três artigos ${ }^{17,19,22}$. Dentre esses profissionais, 

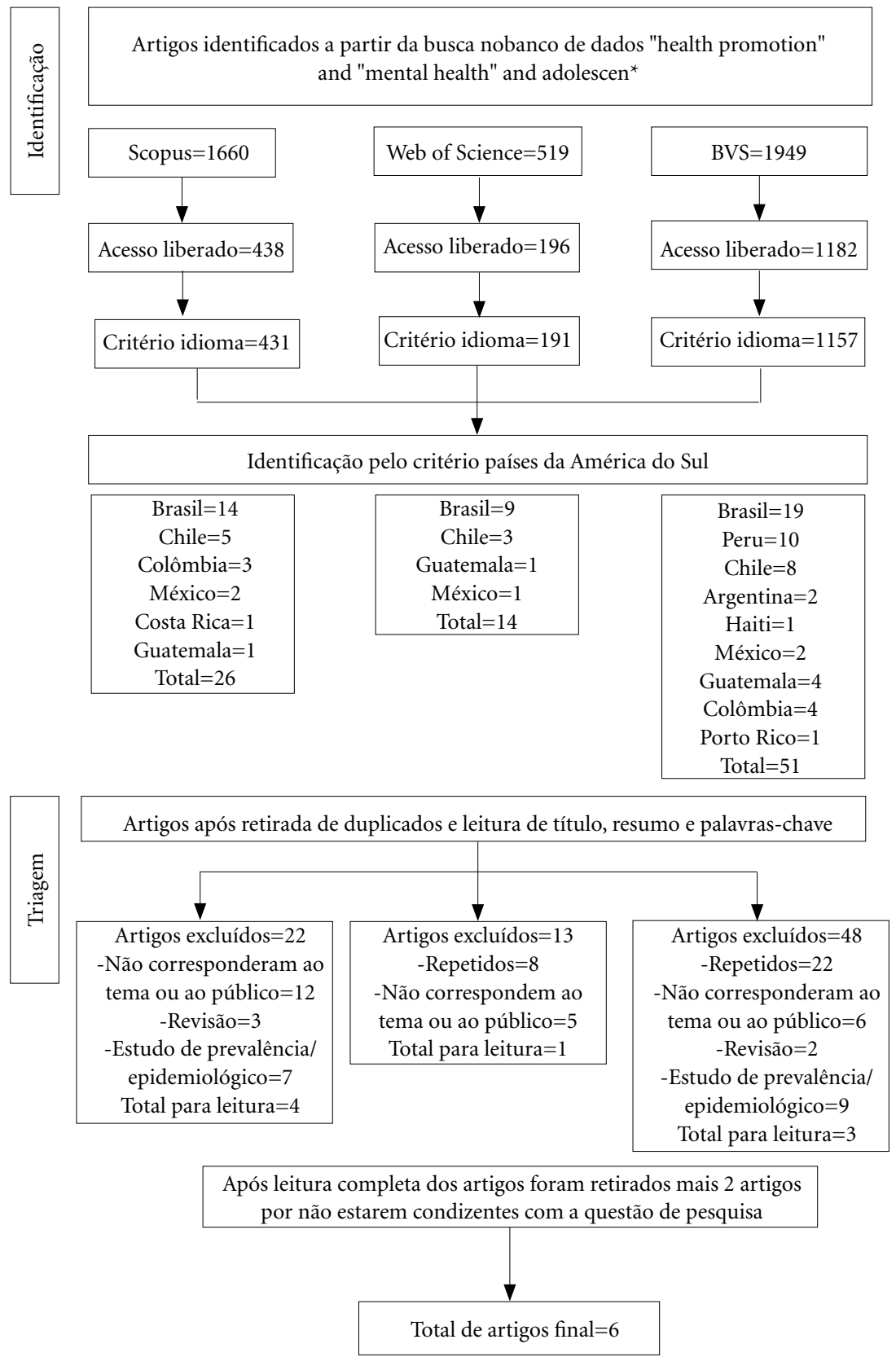

Figura 1. Fluxograma das buscas pelas produções científicas.

Fonte: Elaborado pelos autores.

merece destaque a figura do professor que apareceu em duas dessas ações ${ }^{17,22}$. Essa relevância é justificada nos estudos pelo local estratégico em que a escola se encontra em decorrência do tempo que esses adolescentes permanecem nesse contexto, vinculação e parceria que esse espaço pode representar.
Partindo do contexto territorial escolar para o contexto social, foi observado que em dois dos estudos analisados foram identificados atravessamentos na implementação da PS em decorrência de situações de vulnerabilidade presentes no território ${ }^{17,19}$. São citados, a título de exemplo, a interferência do tráfico nas organizações daquele 
Quadro 1. Identificação dos artigos selecionados.

\begin{tabular}{|l|l|l|l|l|}
\hline \multicolumn{1}{|c|}{ Título } & \multicolumn{1}{|c|}{ Revista } & \multicolumn{1}{c|}{ Autores } & Ano & \multicolumn{1}{c|}{ País } \\
\hline $\begin{array}{l}\text { Adequações do piloto do programa } \\
\text { Unplugged\#Tamojunto para promoção à saúde e } \\
\text { prevenção de drogas em escolas Brasileiras }\end{array}$ & $\begin{array}{l}\text { Ciência e Saúde } \\
\text { Coletiva }\end{array}$ & Pedroso et al..$^{17}$ & 2019 & Brasil \\
\hline $\begin{array}{l}\text { Extensão Acadêmica multiprofissional: experiências na } \\
\text { educação em saúde de jovens em ambiente escolar }\end{array}$ & Revista Univap & Lopes et al. ${ }^{18}$ & 2019 & Brasil \\
\hline $\begin{array}{l}\text { Atenção básica e cuidado colaborativo na atenção } \\
\text { psicossocial de crianças e adolescentes: Facilitadores e } \\
\text { barreiras }\end{array}$ & $\begin{array}{l}\text { Ciência e Saúde } \\
\text { Coletiva }\end{array}$ & Teixeira et al. ${ }^{19}$ & 2017 & Brasil \\
\hline $\begin{array}{l}\text { Conocimiento adquirido y adherencia en un diplomado } \\
\text { sobre la salud mental para docentes orientadores }\end{array}$ & $\begin{array}{l}\text { Revista de salud } \\
\text { pública }\end{array}$ & $\begin{array}{l}\text { Gómez- } \\
\text { Restrepo et al. } .^{22}\end{array}$ & 2014 & Colômbia \\
\hline $\begin{array}{l}\text { Evaluación de la eficacia de un programa preventivo para } \\
\text { la depresión en adolescentes de sexo feminino }\end{array}$ & $\begin{array}{l}\text { Terapia } \\
\text { Psicologica }\end{array}$ & Cova et al. ${ }^{20}$ & 2011 & Chile \\
\hline $\begin{array}{l}\text { Improving physical fitness and emotional well-being in } \\
\text { adolescents of low socioeconomic status in Chile: Results } \\
\text { of a school-based controlled trial }\end{array}$ & $\begin{array}{l}\text { Health } \\
\text { Promotion } \\
\text { International }\end{array}$ & $\begin{array}{l}\text { Bonhauser et } \\
\text { al. }^{21}\end{array}$ & 2005 & Chile \\
\hline
\end{tabular}

Fonte: Elaborado pelos autores.

contexto e violência e consequentemente o receio dos professores em desenvolver ações que possam gerar conflitos e riscos com esse grupo ${ }^{17}$.

Foram observadas ainda, dificuldades relacionadas a rigidez e dificuldades na operacionalização das ações ${ }^{18,20}$. São relatadas impossibilidade de uma atenção especial aos adolescentes que demandaram algo mais individualizado após as intervenções, além da dificuldade ao acesso de equipamentos de multimídia ${ }^{18}$. Foram citadas também como dificultador na implementação a inflexibilidade de horários para a proposição das ações e para atender todas as turmas escolares, e inadequações quanto à infraestrutura do local o que, segundo os autores, prejudicou o desenvolvimento de propostas mais dinâmicas, como a organização em roda dos adolescentes ${ }^{18,20}$.

Como última categoria temática levantada, foi identificado um estudo que aborda sobre as ações na ABS. O mesmo apontou para ausência de ações desenvolvidas neste nível de atenção à saúde, ainda que as ações de promoção à saúde sejam pauta prioritária da $\mathrm{ABS}^{19}$.

\section{Discussão}

A AL apresenta estruturas de financiamento e implementação de programas qualificadas por diferenças regionais. Diante disso, observa-se no contexto latino-americano a coexistência de sistemas de saúde pública que diferem entre si, in- clusive no investimento e valorização da Atenção primária e ações em PS $^{23,24}$. Diante disso, pode-se levantar a hipótese que os investimentos na produção científica sobre a Atenção Primária e suas ações de PS acompanham a significação que a saúde representa para as instâncias governamentais locais e a forma com que os países se organizam politicamente quanto ao desenvolvimento dessas ações, o que poderia justificar a concentração de produção científica nos três países identificados nesta revisão.

De qualquer forma, os resultados do presente estudo, sinalizam para a escassez de produções acadêmicas que focalizam a PS junto à população adolescente na AL. Dos seis Estudos identificados, cinco deles são da última década, o que indica uma possível contemporaneidade científica no que se refere à essa temática. Considerando o panorama mundial, é possível refletir que, embora as discussões sobre a PS tenham se iniciado na década de 1970, somente a última Conferência Mundial de Promoção em Saúde, ocorrida em $2016^{1,25}$, foi realizada em um país latino-americano. Com isso, parece que o campo ainda é incipiente e está em construção, com uma participação e representação possivelmente crescente dos países da AL.

Em relação ao tipo de pesquisa adotado pelos estudos encontrados, foi identificada uma predominância de produções de abordagem experimental e quase experimental, com uma valorização dos processos de avaliação de intervenções 
Quadro 2. Caracterização dos artigos selecionados.

\begin{tabular}{|c|c|c|c|c|c|}
\hline Artigo & $\begin{array}{l}\text { Tipo de } \\
\text { estudo }\end{array}$ & $\begin{array}{c}\text { Fundamentação } \\
\text { teórica }\end{array}$ & Participantes & Como foi desenvolvido & Local \\
\hline 1 & $\begin{array}{l}\text { Estudo } \\
\text { qualitativo }\end{array}$ & $\begin{array}{l}\text {-Teoria } \\
\text { Fundamentada } \\
\text { Construtivista } \\
\text {-Comprehensive } \\
\text { Social Influence } \\
\text { Model } \\
\text {-Teoria de } \\
\text { difusão das } \\
\text { informações }\end{array}$ & $\begin{array}{l}\text { Multiplicadores, } \\
\text { supervisores, } \\
\text { professores, alunos, } \\
\text { pais, comunidade, } \\
\text { profissionais da } \\
\text { saúde e educação. } \\
2161 \text { educandos de } \\
11 \text { a } 14 \text { anos e de } \\
13 \text { a } 14 \text { anos. }\end{array}$ & $\begin{array}{l}\text { O programa \#Tamojunto } \\
\text { é organizado em } 12 \text { aulas } \\
\text { conduzidas por professores e } 3 \\
\text { oficinas envolvendo profissionais } \\
\text { da saúde, pais e comunidade. }\end{array}$ & $\begin{array}{l}8 \text { Escolas } \\
\text { públicas }\end{array}$ \\
\hline 2 & $\begin{array}{l}\text { Relato de } \\
\text { experiência }\end{array}$ & $\begin{array}{l}\text { Educação em } \\
\text { saúde }\end{array}$ & $\begin{array}{l}\text { Adolescentes } \\
\text { de } 14 \text { a } 19 \text { anos } \\
\text { estudantes do } 1^{\circ} \text {, } \\
2^{\circ} \text { e } 3^{\circ} \text { do ensino } \\
\text { médio. }\end{array}$ & $\begin{array}{l}\text { Educação em saúde por meio } \\
\text { de rodas de conversa, dinâmicas } \\
\text { e jogos sobre diferentes temas, } \\
\text { dentre eles a saúde mental. } \\
\text { Foram realizados seis encontros e } \\
\text { uma palestra final }\end{array}$ & $\begin{array}{l}1 \text { Escola } \\
\text { estadual }\end{array}$ \\
\hline 3 & $\begin{array}{l}\text { Estudo } \\
\text { exploratório, } \\
\text { de } \\
\text { abordagem } \\
\text { qualitativa }\end{array}$ & $\begin{array}{l}\text {-Pesquisa Social } \\
\text { em Saúde } \\
\text {-Promoção da } \\
\text { Saúde } \\
\text {-Educação em } \\
\text { Saúde }\end{array}$ & $\begin{array}{l}25 \text { trabalhadores } \\
\text { da ESF e um } \\
\text { representante } \\
\text { do CAPSi por } \\
\text { encontro }\end{array}$ & $\begin{array}{l}\text { Total de duas fases: } \\
\text { 1) Levantamento dos } \\
\text { encaminhamentos para o CAPSi; } \\
\text { 2) grupos focais e de intervenção } \\
\text { com os profissionais de saúde, } \\
\text { em três etapas }\end{array}$ & $\begin{array}{l}\text { Serviços de } \\
\text { saúde (Atenção } \\
\text { Básica e } \\
\text { CAPSi) }\end{array}$ \\
\hline 4 & $\begin{array}{l}\text { Estudo } \\
\text { quase- } \\
\text { experimental }\end{array}$ & Não especificada & $\begin{array}{l}\text { Conselheiros } \\
\text { escolares e } \\
\text { Professores que } \\
\text { trabalham com } \\
\text { crianças de } 6 \text { a } 11 \\
\text { anos e adolescentes } \\
\text { de } 12 \text { anos a } 18 \\
\text { anos. Total de } \\
234 \text { crianças e } \\
\text { adolescentes }\end{array}$ & $\begin{array}{l}\text { Os professores passaram por } \\
\text { um processo de capacitação } \\
\text { em uma plataforma virtual. } \\
\text { Também ocorreram duas sessões } \\
\text { presenciais }\end{array}$ & $\begin{array}{l}\text { Escolas de } \\
\text { Boyacá e } \\
\text { Cundinamarca }\end{array}$ \\
\hline 5 & $\begin{array}{l}\text { Estudo } \\
\text { quase- } \\
\text { experimental }\end{array}$ & $\begin{array}{l}\text {-Coping } \\
\text {-Cognitivo- } \\
\text { comportamental }\end{array}$ & $\begin{array}{l}\text { Adolescentes do } \\
\text { sexo feminino. } \\
\text { 1. Amostra: } 101 \\
\text { adolescentes no } \\
\text { grupo intervenção } \\
\text { e } 108 \text { no controle } \\
\text { 2. Amostra: } \\
119 \text { meninas } \\
\text { para o grupo } \\
\text { experimental e } 118 \\
\text { participantes para } \\
\text { o controle. }\end{array}$ & $\begin{array}{l}11 \text { sessões de } 1 \text { h30 de duração } \\
\text { em grupos de } 15 \text { a } 23 \text { pessoas. O } \\
\text { programa foi desenvolvido para } \\
\text { que os adolescentes entendessem } \\
\text { a relação entre emoções, } \\
\text { cognições e comportamentos } \\
\text { e fortalecessem uma série de } \\
\text { habilidades. Foram criados } \\
\text { espaços para analisar e colocar } \\
\text { em prática essas habilidades em } \\
\text { relação aos problemas habituais } \\
\text { da adolescência. }\end{array}$ & $\begin{array}{l}9 \text { Escolas } \\
\text { municipal e } \\
\text { particular }\end{array}$ \\
\hline 6 & $\begin{array}{l}\text { Estudo } \\
\text { quase- } \\
\text { experimental }\end{array}$ & $\begin{array}{l}\text {-Abordagem de } \\
\text { aprendizagem } \\
\text { de adultos }\end{array}$ & $\begin{array}{l}198 \text { estudantes de } \\
15 \text { anos cursando o } \\
9^{\circ} \text { ano }\end{array}$ & $\begin{array}{l}\text { Para o grupo de intervenção foi } \\
\text { proposto três vezes por semana } \\
\text { sessões de atividades físicas de } 90 \\
\text { minutos que variavam conforme } \\
\text { o gênero. Para o grupo controle } \\
\text { as atividades físicas ocorriam } \\
\text { uma vez por semana }\end{array}$ & $\begin{array}{l}1 \text { Escola } \\
\text { pública }\end{array}$ \\
\hline
\end{tabular}


Quadro 3. Eixos temáticos identificadas nos artigos selecionados.

\begin{tabular}{|l|r|}
\hline Eixos temáticos & \multicolumn{1}{|c|}{ Artigos } \\
\hline Dificuldade nas parcerias intersetoriais & $1,2,3,5$ \\
\hline $\begin{array}{l}\text { Potência das ações em grupo com } \\
\text { adolescentes }\end{array}$ & $2,5,6$ \\
\hline $\begin{array}{l}\text { Importância e necessidade de investir na } \\
\text { formação profissional }\end{array}$ & $1,3,4$ \\
\hline $\begin{array}{l}\text { Dificuldade em implementar estratégias } \\
\text { devido a vulnerabilidade social }\end{array}$ & 1,3 \\
\hline $\begin{array}{l}\text { Rigidez e dificuldades operacionais que } \\
\text { limitam as ações }\end{array}$ & 2,5 \\
\hline $\begin{array}{l}\text { Ações de cuidado são raras ou } \\
\text { inexistentes na ABS }\end{array}$ & 3 \\
\hline
\end{tabular}

Fonte: Elaborado pelos autores.

pré-estabelecidas por aqueles que a "aplicam", o que representa uma forma tradicional de produzir conhecimento. Além disso, os estudos identificados apresentaram, em suas discussões, conversas com referenciais europeus e norte-americanos. Nenhum dos estudos encontrados utilizou de abordagem participativa, por exemplo, ou realizou um levantamento das demandas dos contextos-alvo no desenvolvimento das estratégias de promoção à saúde, fatores que têm sido debatidos enquanto relevantes na elaboração de ações desse tipo, ou seja, que fomentem a participação ativa da comunidade e favoreçam construções autônomas e singulares para determinados contextos ${ }^{4}$.

Somado a isso, existe a própria desvalorização histórica do saber do adolescente, seu recente reconhecimento legal, sua estigmatização e exclusão dos processos de construção de conhecimento $^{26}$. Rossi et al. ${ }^{7}$ apontam ainda sobre a falta de produção científica na saúde mental COM os adolescentes e não SOBRE essa população, favorecendo desta maneira uma construção mais prática do conhecimento, realística e possível de provocar efetivamente transformações dentro das micropolíticas das relações, no caso, práticas de PS mais significativas e efetivas.

Em se tratando do local do desenvolvimento das pesquisas encontradas, observa-se que a escola foi o ambiente focalizado em cinco estudos. A escola representa, por meio de seus mecanismos de funcionamento uma perspectiva psicossocial que pode auxiliar na participação social, além dos próprios processos de cidadania, e é entendida como espaço promotor de saúde em decorrência desse potencial de possibilitar formas de existência no mundo e novas possibilidades de sociabilidade $^{27}$.

Vieira et al. ${ }^{28}$, define, ainda, que as escolas são mais acessíveis do que os serviços de saúde e oportunizam aos adolescentes, intervenções que sejam menos estigmatizadoras, além disso, reforça-se sobre a baixa procura por serviços de saúde por esta faixa-etária ${ }^{12}$. Portanto, o desenvolvimento de ações em contextos que os adolescentes já estão e frequentam pode facilitar o desenvolvimento de ações direcionadas a eles, incluindo as de PS.

Nessa direção, a parceria entre os setores saúde e educação, mostrou-se um potencial a ser explorado em ações de PS com esta faixa etária, tendo em vista o desenvolvimento de práticas intersetoriais, as quais vão além de usar o contexto da escola unicamente como um meio de acesso à população-alvo, mas também como um parceiro no desenvolvimento de ações partilhadas e desenhadas com e para esta população. Nessa perspectiva, outros setores da assistência também podem ser convidados a compor, na medida em que a intersetorialidade se apresenta como eixo conceitual da PS, visando o desenvolvimento de ações efetivas e condizentes com as realidades práticas ${ }^{29}$.

Cid et at. ${ }^{30}$, tratando das possibilidades da terapia ocupacional em ações de saúde mental no contexto escolar, afirmam que práticas de PS nesse contexto, não são clínicas, mas de natureza intersetorial, sendo disparadas a partir da construção colaborativa do cuidado, ou seja, trata-se de um trabalho desenvolvido com educadores, familiares e a própria comunidade, visando à garantia de acesso e inclusão à escola.

Ainda, considerando a articulação da saúde com outros setores, nenhum estudo foi encontrado analisando o setor arte-cultura como possibilitador de prevenção e promoção em saúde mental, considerando o aspecto estratégico do campo artístico-cultural para a atenção à saúde mental e o caráter "extremamente promissor" que essas iniciativas podem desempenhar ${ }^{31}$. Além disso, em se tratando dos contextos Latino-Americanos, destaca-se a importância do aspecto cultural como um importante pilar para o desenvolvimento da identidade, processos criativos e resistência contra a desigualdade social, sendo essencial para o desenvolvimento e consolidação da independência da identidade das nações e bem estar ${ }^{32}$. Ações dessa natureza, quando voltadas para juventude podem promover empoderamentos tanto do âmbito individual quando coletivo, podendo causar reflexões sobre a forma de existência no mundo ${ }^{33}$. Quando se fala em saúde 
mental infantojuvenil e ações de saúde mental por meio da arte e cultura, as produções científicas apresentam-se incipientes, principalmente quando se trata de uma perspectiva mais salutogênica em saúde ${ }^{34}$.

Em relação às abordagens teóricas selecionadas como norteadoras das intervenções, é importante destacar a escolha predominante dos estudos pela abordagem comportamental-cognitiva de aspecto preventivo encontrados nesta revisão. A escolha por essa perspectiva, que põe foco no sujeito mostra-se em conformidade com outros estudos desenvolvidos na $\mathrm{AL}^{3}$. Tratando-se ainda especificamente do contexto latino-americano, africano e asiático, observa-se que esses países ao tentarem se encaixar nas agendas universais de PS, acabam presos a aspectos mais normativos, conservadores e burocráticos, inclusive na produção de conhecimento, já que as propostas de ações de PS realizadas pelos organismos internacionais ou ainda como em países como o Canadá não aprofundam em questões como singularidades de contextos em situação de vulnerabilidade e injustiça ${ }^{24}$.

Nesta direção, identifica-se uma diferença de perspectiva dos estudos brasileiros encontrados que relataram ações voltadas para Educação em Saúde. Se por um lado a educação em saúde é importante pelo conteúdo informativo em si, “informacional-global", por outro é necessário provocar sobre a ampliação dessa estratégia, concordante com o que a PS tem desenvolvido em sua corrente mais progressista, ou seja, de promover um "comunicacional-local" de modo a romper com as "monoculturas do saber" 24 .

[...] o enfoque educacional, tão fundamental nos processos de promoção da saúde e prevenção de agravos, não pode ficar preso às tendências modeladoras, fortemente difundidas com base no paradigma comportamentalista. A atitude construtivista é aquela que melhor parece concorrer para que as pessoas possam de fato buscar e se apropriar de informações que façam sentido para elas, se mobilizar autenticamente e achar as alternativas práticas que permitam superar as situações que as vulnerabilizam. ${ }^{35}$ (p.140).

Identifica-se a importância, dentro da saúde, da ferramenta da informação como meio de construção de processos de saúde, utilizando-se para isso da educação em saúde e da cognitivocomportamental, onde o profissional da saúde seria um facilitador da transmissão de um conhecimento específico. O que é questionado é a limitação que esses tipos de estratégias podem esbarrar ao desconsiderar ou subestimar os aspectos subjetivos que podem influenciar nas tomadas de decisão. Além disso, a própria característica modeladora do comportamento que o risco pode representar em abordagens como essas, funcionando de maneira deturpada como uma tecnologia moral e não propriamente produtora de saúde ${ }^{36}$.

Dentre as estratégias de intervenção foi identificado certa "similaridade" no modo como essas ações foram desenvolvidas, utilizando de rodas de conversa, palestras e oficinas. A utilização de grupos demonstra, por meio da análise dos resultados dos artigos encontrados, ser uma estratégia interessante para ações de promoção em saúde mental para adolescentes, além de baixo custo, o que, para países da AL pode representar um importante facilitador.

Outra estratégia encontrada pelos estudos, identificada como facilitadora das ações, foi em relação ao investimento na formação profissional que trabalham com esta faixa-etária, em especial os professores que em geral apresentam um bom acesso aos adolescentes. É importante frisar que a formação dos educadores sobre processos de saúde mental representa uma estratégia de baixo custo e pode influenciar a longo prazo na diminuição dos aspectos estigmatizantes relacionados a esse público ${ }^{30}$.

Cid et al. ${ }^{30}$ apontam que há pouca informação disponível sobre a formação destinada aos docentes em relação à saúde mental infantojuvenil, gerando insegurança nesses profissionais e dificuldades no manejo de situações que aparecem relacionadas aos jovens com sofrimento psíquico. Nesse sentido, as ações intersetoriais poderiam contribuir com a troca de saberes, envolvendo dessa maneira diferentes atores e serviços, de modo a orientar e garantir integralidade no cuidado.

Dentre os desafios das ações de PS direcionada ao público adolescente, os estudos encontrados levantaram sobre as fragilidades estruturais para operacionalização das ações relacionadas a vulnerabilidades dos contextos-alvo. Sobre isso, Souza et al. ${ }^{37}$ afirmam que iniquidade social é o fator de maior impacto na saúde humana. Estar em um contexto de vulnerabilidade significaria, portanto, acesso limitado às possibilidades materiais, simbólicas e culturais, ou seja, uma população nesse contexto vive à margem das possibilidades sociais e pode ter impactos diretos no desenvolvimento físico, cognitivo, emocional e ocupacional.

Além disso, a não flexibilidade de horários e de uma organização mais democrática, incluindo os participantes nesse processo, representam um 
dificultador que poderia ser solucionado com o aumento da comunicação e a inclusão de parcerias mais estruturadas entre os setores. Sugere-se a partir desses resultados que a atenção básica considere de maneira permanente a colaboração com as escolas através de ações contínuas e estruturadas no próprio cronograma anual escolar.

Segundo Lancetti ${ }^{38}$, para a saúde mental a concepção de complexidade da atenção é invertida, o nível primário representaria a complexidade, na medida em que se trabalha com a família, rede de apoio, atores sociais e o próprio território. Pensar, portanto, em ações de PS, no campo da saúde mental, na atenção primária, perpassa uma complexidade com urgência a ser discutida.

\section{Conclusão}

Os resultados do presente estudo identificam uma lacuna na literatura referente às produções de PS de adolescentes na AL. Além disso, foi possível observar ausência de propostas que levem em consideração as demandas locais e a participação dos diferentes atores, incluindo os adolescentes, na definição das estratégias propostas, em diálogo com o que tem sido produzido mundialmente no campo das PS. Não foram encontrados estudos que contemplassem o setor arte-cultural para a produções dessas ações. A escola foi o principal contexto das ações, o que sinaliza para o potencial deste ambiente para o desenvolvimento de estratégias significativas de PS. Sinaliza-se, ainda, que intervenções do tipo grupal e que auxiliem profissionais que lidam com adolescentes de maneira direta pode ser uma estratégia de baixo custo para os países latino-americanos.

\section{Colaboradores}

TT Souza participou do processo de concepção do estudo, identificação dos estudos, na análise/ interpretação de dados e elaboração do texto. AC Almeida participou da identificação dos estudos da revisão e análise/interpretação dos dados. ADSA Fernandes da análise/interpretação dos dados, elaboração do texto e revisão do manuscrito. MFB Cid participou da concepção do estudo elaboração do texto e revisão do manuscrito. 


\section{Referências}

1. Buss PM. Uma introdução ao Conceito de Promoção da Sáude. In: Czeresnia D, Freitas CM, editores. Promoção da saúde: conceitos, reflexões, tendências. Rio de Janeiro: SciELO-Editora Fiocruz; 2009. p. 19-42.

2. Spink MJP. Psicologia Social e Saúde: trabalhando com a complexidade. Quaderns Psicol 2010; 12(1):4156.

3. Borghi CMSO, Oliveira RM, Sevalho G. Determinação ou Determinantes Sociais da Saúde: Texto e Contexto na América Latina. Trab Educ Saúde 2018; 16(3):869-897.

4. Freitas CM. A vigilância da Saúde para a Promoção da Saúde. In: Czeresnia D, Freitas CM, editores. Promoção da saúde: conceitos, reflexões, tendências. Rio de Janeiro: SciELO-Editora Fiocruz; 2009. p. 145-164.

5. Acosta A. O Bem Viver: uma alternativa ao desenvolvimento. In: Costa A, editor. O Bem Viver: uma oportunidade para imaginar outros mundos. São Paulo: Elefante Editora; 2016. p. 69-88.

6. Organização Mundial da Saúde (OMS). Global Accelerated Action for the Health of Adolescents (AA-HA!): Guidance to Support Country Implementation. Genebra: OMS; 2017.

7. Rossi LM, Marcolino TQ, Speranza M, Cid MFB. Crise e saúde mental na adolescência: a história sob a ótica de quem vive. Cad Saude Publica 2019; 35(3):1-12.

8. Fatori D, Brentani A, Grisi SJFE, Miguel EC, Graeff -Martins AS. Prevalência de problemas de saúde mental na infância na atenção primária. Cien Saude Colet 2018; 23(9):3013-3020.

9. Patel V, Flisher AJ, Nikapota A, Malhotra S. Promoting child and adolescent mental health in low and middle income countries. J Child Psychol Psychiatry 2008; 49(3):313-334.

10. Saxena S, Paraje G, Sharan P, Karam G, Sadana R. The 10/90 divide in mental health research: trends over a 10-year period. Br J Psychiatry 2006; 188(1):81-82.

11. Schmidt CK, Raque-Bogdan TL, Piontkowski S, Schaefer KL. Putting the positive in health psychology: A content analysis of three journals. J Health Psychol 2011; 16(4):607-620.

12. Fernandes ADSA. Cuidado em Saúde Mental Infantojuvenil na Atenção Básica à Saúde: práticas, desafios e perspectivas. 2015-2019 [tese]. São Carlos: Universidade Federal de São Carlos; 2019.

13. Barata RB. Políticas para o enfrentamento das desigualdades. In: Barata RB, editor. Como e por que as desigualdades sociais fazem mal à saúde. Rio de Janeiro: Editora Fiocruz; 2009. p. 95-108.

14. Mendonça MHM. O desafio da política de atendimento à infância e à adolescência na construção de políticas públicas eqüitativas. Cad Saude Publica 2002; 18:S113-S120.

15. Broome ME. Integrative literature reviews for the development of concepts. Concept development in nursing: foundations, techniques and applications. Philadelphia: WB Saunders Company; 2000.

16. Mendes KDS, Silveira RCCP, Galvão CM. Revisão integrativa: método de pesquisa para a incorporação de evidências na saúde e na enfermagem. Texto Contexto Enferm 2008; 17(4):758-764.
17. Pedroso RT, Hamann EM. Adequações do piloto do programa Unplugged\#Tamojunto para promoção à saúde e prevenção de drogas em escolas brasileiras. Cien Saude Colet 2019; 24(2):371-381.

18. Lopes RNS, Takeshita IM, Freire AFS, Dias AAS. Extensão acadêmica multiprofissional: experiências na educação em saúde de jovens em ambiente escolar. Rev Univap 2019; 25(48):92-103.

19. Teixeira MR, Couto MCV, Delgado PGG. Atenção básica e cuidado colaborativo na atenção psicossocial de crianças e adolescentes: facilitadores e barreiras. Cien Saude Colet 2017; 22(6):1933-1942.

20. Cova F, Rincón P, Melipillán R. Evaluación de la eficacia de un programa preventivo para la depresión en adolescentes de sexo femenino. Terapia Psicol 2011; 29(2):245-250.

21. Bonhauser M, Fernandez G, Püschel K, Yañez F, Montero J, Thompson B, Coronado G. Improving physical fitness and emotional well-being in adolescents of low socioeconomic status in Chile: results of a school-based controlled trial. Health Promot Int 2005; 20(2):113-122

22. Gómez-Restrepo C, Tamayo-Martínez N, Ayala-Corredor C, Carrasco-Gil A, Padilla-Muñoz A, Garzón DF, Borda JP, Verhelst P, Ramirez MP, González LM, Gil JF. Conocimiento adquirido y adherencia en un diplomado sobre la salud mental para docentes orientadores. Rev Salud Publica 2014; 16(6):821-833.

23. Cortes FM, Coelho TCB, Sanabria CAP. Seguridade social na América Latina e financiamento da atenção primária de saúde. JMPHC 2019; 11(Supl. 1):e32s.

24. Porto MFS, Pivetta F. Por uma promoção da Saúde Emancipatória em Territórios Urbanos Vulneráveis. In: Czeresnia D, Freitas CM, editores. Promoção da saúde: conceitos, reflexões, tendências. Rio de Janeiro: SciELO-Editora Fiocruz; 2009. p. 207-229.

25. Associação Brasileira de Saúde Coletiva (ABRASCO). $22^{a}$ Conferência Mundial de Promoção da Saúde [Internet]. 2016 [acessado 2020 jan 17]. Disponível em https://www.abrasco.org.br/site/noticias/saude-da -populacao/22a-conferencia-mundial-de-promocaoda-saude/13855/.

26. Fernandes ADSA, Matsukura TS. Adolescentes inseridos em um CAPSi: alcances e limites deste dispositivo na saúde mental infantojuvenil. Temas Psicol 2016; 24(3):977-990.

27. Tano BL, Hayashi MCPI. Saúde mental infantojuvenil e educação: análise bibliométrica da produção científica nacional e internacional (1968-2014). RECIIS 2015; 9(3):1-26.

28. Vieira MA, Estanislau GM, Bressan RF, Bordin IA. Saúde Mental na Escola. In: Estanislau G, Bressan R, editores. Saúde mental na escola. Porto Alegre: Artmed; 2014. p. 13-23.

29. Rivera FJU, Artmann E. Promoção da Saúde e Planejamento Estratégico Situacional: intersetorialidade na busca de maior governabilidade. In: Czeresnia D, Freitas CM, editores. Promoção da saúde: conceitos, reflexões, tendências. Rio de Janeiro: SciELO-Editora Fiocruz; 2009. p. 183- 206. 
30. Cid MFB, Squassoni CE, Gasparini DA, Fernandes LHO. Saúde mental infantil e contexto escolar. Pro -Posi 2019; 30:e20170093.

31. Amarante P, Freitas F, Nabuco ES, Pande MR. Da arteterapia nos serviços aos projetos culturais na cidade: a expansão dos projetos artístico-culturais da saúde mental no território. In: Amarante P, Nocan F, editores. Saúde Mental e Arte: Práticas, saberes e Debates. São Paulo: Zagadoni; 2012. p. 23-38.

32. Organização das Nações Unidas para a Educação, a Ciência e a Cultura (UNESCO). Declaración de México sobre las políticas culturales. conferencia mundial sobre las políticas culturales [Internet]. 1982 [acessado 2020 jan 17]. Disponível em: https://culturalrights. net/descargas/drets_culturals400.pdf.

33. Silva CR, Cardinalli I, Silvestrini MS, Farias AZ, Prado ACSA, Ambrosio L, Oliveira MT, Paula BM. La terapia ocupacional y la cultura: miradas a la transformación social. Rev Chilena Terapia Ocupacional 2017; 17(1):105-113

34. Zarobe L, Bungay $\mathrm{H}$. The role of arts activities in developing resilience and mental wellbeing in children and young people a rapid review of the literature. Perspect Public Health 2017;137(6):337-347.

35. Ayres JRCM, França Júnior I, Calazans GJ, Saletti Filho HC. O conceito de Vulnerabilidade e as práticas de Saúde: novas perspectivas e desafios. In: Czeresnia D, Freitas CM, editores. Promoção da saúde: conceitos, reflexões, tendências. Rio de Janeiro: SciELO-Editora Fiocruz; 2009. p. 121-144
36. Castiel LD, Dardet CA. Discursos sobre riscos e comportamentos Sadios Baseados em Evidências. In: Castiel LD, Dardet CA, editores. A saúde persecutória: os limites da responsabilidade. Rio de Janeiro: SciELO-Editora Fiocruz; 2007. p. 25-34.

37. Souza LB, Panúncio-Pinto MP, Fiorati RC. Crianças e adolescentes em vulnerabilidade social: bem-estar, saúde mental e participação em educação. Cad Bras Terapia Ocupacional 2019; 27(2):251-269.

38. Lancetti A. Clínica peripatética. São Paulo: Hucitec; 2006

Artigo apresentado em 30/05/2020

Aprovado em 31/03/2021

Versão final apresentada em 02/04/2021

Editores-chefes: Romeu Gomes, Antônio Augusto Moura da Silva 